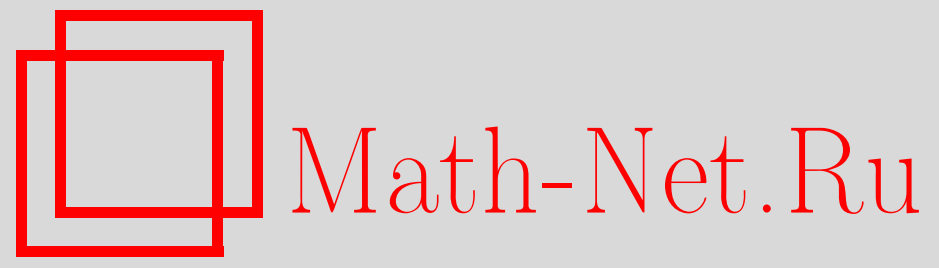

Д. Н. Бабин, Эффективная проверяемость полноты систем автоматных функций с полной булевой частью, Дискрет. матем., 2003, том 15, выпуск 1, 110-130

DOI: https://doi.org/10.4213/dm188

Использование Общероссийского математического портала Math-Net.Ru подразумевает, что вы прочитали и согласны с пользовательским соглашением http://www . mathnet.ru/rus/agreement

Параметры загрузки:

IP : 54.162 .127 .20

26 апреля 2023 г., 11:24:43 


\title{
Эффективная проверяемость полноты систем автоматных функций с полной булевой частью
}

\author{
๑) 2003 г. Д. Н. Бабин
}

\begin{abstract}
Рассматриваются автоматные базисы с полной булевой частью. Построен алгоритм для проверки полноты таких базисов и получены верхние оценки его сложности.
\end{abstract}

\section{1. Введение}

Известно, что решение задачи о полноте относительно операций суперпозиции и обратной связи для систем автоматных функщий наталкивается на существенные трудности. Так в [1] установлена континуальность всякой критериальной системы для этой задачи, а позднее в [2] установлена ее алгоритмическая неразрешимость для конечных систем автоматных функций. Вместе с тем, для спещиальных систем автоматов, выдающих номер своего состояния (автоматов Медведева) и содержащих все булевы функции, указанная задача алгоритмически разрешима [3]. Ранее, в [4] было показано, что при наличия булевых функций алгоритмически разрешима проблема полноты и для произвольных систем автоматов и даже не все булевы функции для этого нужны $[5,6,7]$. Возникший при этом аппарат, сохраняемых предикатов на экспериментах с автоматами, позволил упростить доказательство из статьи [4]. Новый вариант доказательства приводится в настоящей работе.

\section{2. Основные понятия и результаты}

Пусть $E_{2}=\{0,1\}, E_{2}^{\infty}-$ множество всех сверхслов $a(1) a(2) \ldots$, где $a(j) \in E_{2}$, $j=1,2, \ldots$ Пусть

$$
f:\left(E_{2}^{\infty}\right)^{n} \rightarrow\left(E_{2}^{\infty}\right)^{m}
$$

- автоматная функция (а.-функщия), то есть функция, задаваемая рекуррентно соотношениямн

$$
\begin{aligned}
q(1) & =q_{1}, \\
q(t+1) & =\varphi\left(q(t), a_{1}(t), \ldots, a_{n}(t)\right), \\
b_{j}(t) & =\psi_{j}\left(q(t), a_{1}(t), \ldots, a_{n}(t)\right), \quad j=1, \ldots, m .
\end{aligned}
$$

где $q \in Q=\left\{q_{1}, \ldots, q_{r}\right\}$. 
Параметр $q$ называется состоянием а.-функция $f, q_{1}$ - ее начальным состоянием, векторы $a=\left(a_{1}, \ldots, a_{n}\right)$ и $b=\left(b_{1}, \ldots, b_{m}\right)$ называются входной и выходной буквами, а сверхслова $a(1) a(2) \ldots$ и $b(1) b(2) \ldots$ - входным и выходным сверхсловами, соответственно. Класс всех а.-функций обозначим $P_{a}$. Если $|Q|=1$, то автоматную функию (1) можно отождествить с булевой вектор-функцией $\psi_{j}\left(q(t), a_{1}(t), \ldots, a_{n}(t)\right)$ и считать булевы функции частным случаем автоматных. Известно, что все булевы функции порождаются одной функцией $\overline{\boldsymbol{x}} \vee \bar{y}$.

В классе автоматных функций введем операции суперпозиции и обратной связи. Для суперпозиция будем использовать операции

$$
\begin{aligned}
(\eta f)\left(x_{1}, \ldots, x_{n}\right) & =f\left(x_{2}, x_{3}, \ldots, x_{n}, x_{1}\right), \\
(\varepsilon f)\left(x_{1}, \ldots, x_{n}\right) & =f\left(x_{2}, x_{1}, x_{3}, \ldots, x_{n}\right), \\
(\Omega f)\left(x_{1}, \ldots, x_{n-1}\right. & =f\left(x_{1}, x_{1}, x_{2}, \ldots, x_{n-1}\right), \\
(\nabla f)\left(x_{1}, x_{2}, \ldots, x_{n+1}\right) & =f\left(x_{2}, x_{3}, \ldots, x_{n+1}\right), \\
(f * g)\left(x_{1}, x_{2}, \ldots, x_{l+n-1}\right) & =f\left(g\left(x_{1}, \ldots, x_{l}\right), x_{l+1}, \ldots, x_{l+n-1}\right) .
\end{aligned}
$$

Операция обратной связи, примененная к $\boldsymbol{i}$-й входной и $\boldsymbol{j}$-й выходной переменным а.-функции $f\left(x_{1}, \ldots, x_{n}\right)=\left(y_{1}, \ldots, y_{m}\right)$, задает а.-функцию

$$
g\left(x_{1}, \ldots, x_{i-1}, x_{i+1}, \ldots, x_{n}\right)=\left(y_{1}, \ldots, y_{i-1}, y_{i+1}, \ldots, y_{m}\right),
$$

вычисляемую алгоритмически следующим образом. Считаем, что обратная связь применима к $f$ в состояния $q$, если $\psi_{j}$ в уравнении (1) фиктивно зависит от $a_{i}$ при $q(t)=q$, а вычисление $b_{s}(t)$ осуществляется по схеме

$$
\begin{aligned}
q(1)= & q_{1}, \\
q(t+1)= & \varphi\left(q(t), a_{1}(t), \ldots, a_{i-1}(t), \psi_{j}\left(q(t), a_{1}(t), \ldots, a_{i-1}(t),\right.\right. \\
& \left.\left.a_{i+1}(t), \ldots, a_{n}(t)\right), a_{i+1}(t), \ldots, a_{n}(t)\right), \\
b_{s}(t)= & \psi_{s}\left(q(t), a_{1}(t), \ldots, a_{i-1}(t), \psi_{j}\left(q(t), a_{1}(t), \ldots, a_{i-1}(t),\right.\right. \\
& \left.\left.a_{i+1}(t), \ldots, a_{n}(t)\right), a_{i+1}(t), \ldots, a_{n}(t)\right), \quad s=1,2, \ldots, j-1, j+1, \ldots, m .
\end{aligned}
$$

Считаем, что обратная связь применима к $f$, если она применима в начальном состоянии $q_{1}$ и из ее применимости в состоянии $q(t)$ следует применимость в состоянии $q(t+1)$. Пусть $M \subseteq P_{a}$, обозначим через $[M]$ множество всех а.-функций, получающихся из $M$ с помощью операций суперпозиции и обратной связи. Множество $M$ называется полным, если $[M]=P_{a}$. Проблема полноты для $P_{a}$ состоит в описании всех полных множеств $M$.

Справедлива следующая теорема.

Теорема 1. Для конечной системы $M \subset P_{a}$ равенство

$$
[M \cup\{\bar{x} \vee \bar{y}\}]=P_{a}
$$

алгоритмически проверяемо, и число шагов проверки не превосходит $\left(2^{2^{|Q|^{2}+1}}\right) !$

\section{3. Основные леммы и доказательство теоремы}

Обозначим

$$
\bar{X}, \quad \mathbf{1}, \quad \mathbf{0}, \quad f_{\text {sh }}, \quad f_{\&}, \quad f_{\vee}
$$


автоматные интерпретации истинностных функций, соответственно,

$$
\bar{x}, \quad 1, \quad 0, \quad \bar{x}_{1} \vee \bar{x}_{2}, \quad x_{1} \& x_{2}, \quad x_{1} \vee x_{2} .
$$

Для натуральных $D, N$ автоматную функцию с уравнениями (1), где

$$
m=n=D+N, \quad Q=\{1, \ldots, D+N\},
$$

и для любого $a \in E_{2}^{D+N}$

$$
\begin{aligned}
& \psi(i, a)=0^{i-1} 10^{D+N-i}, \\
& \varphi(i, a)= \begin{cases}i+1 & \text { при } i<D+N, \\
D+1 & \text { при } i=D+N,\end{cases}
\end{aligned}
$$

назовем $(D, N)$-счетчиком и обозначим через $B_{D, N} ; B_{D, N}$ выдает всегда одну и ту же последовательность с периодом $N$ и предпериодом $D$. Если при тех же условиях

$$
\psi\left(i,\left(a_{1}, \ldots, a_{D+N}\right)\right)=a_{i},
$$

то такую автоматную функщию назовем $(D, N)$-селектором и обозначим через $C_{D, N}$. Множество всех счетчиков обозначим через $\mathbf{K}$.

Без ограничения общности, будем исследовать на полноту системы вида $\left\{f_{\mathrm{sh}}, f\right\}$, где $f$ задается уравнениями (1).

Для $D, 0 \leqslant D \leqslant s$, последовательность,

$$
(a(1), b(1)),(a(2), b(2)), \ldots,(a(s), b(s)),
$$

где

$$
\begin{aligned}
& a(1), \ldots, a(s) \in E_{2}^{n}, \quad q(1), \ldots, q(s+1) \in Q, \quad b(1), \ldots, b(s) \in E_{2}^{m}, \\
& b(i)=\psi(q(i), a(i)), \quad q(i+1)=\varphi(q(i), a(i)), \quad i=1, \ldots, s, \\
& q(1)=q_{1}, \quad \varphi(q(s), a(s))=q(D+1),
\end{aligned}
$$

назовем $(D, s)$-экспериментом с а.-функцией $f$.

Пусть $i, j$ - натуральные числа, $1 \leqslant i, j \leqslant s, j \neq i$. Скажем, что а.-функция $f$ является $(j, i)$-зависимой, если для каждой последовательности (2) выполнено соотношение

$$
\psi(q(j), a(i))=b(i))
$$

Автоматная функция $f$ допускает счетчик $B_{D, N}$, если для некоторого $s=D+k N$ и любых $i \neq j, 1 \leqslant i, j \leqslant s$, а.-функщия $f$ не является $(j, i)$-зависимой.

Справедливы следующие леммы.

Лемма 1. Включение $\left[\left\{f, f_{\mathrm{sh}}\right\}\right] \supseteq \mathbf{K}$ справедливо тогда и только тогда, когда для любого натурального $N$ найдутся $D, s=D+k N$ такие, что $f$ допускает счетчик $B_{D, N}$.

Лемма 2. Отночение включения $\left[\left\{f, f_{\mathrm{sh}}\right\}\right] \supseteq \mathbf{K}$ алгоритмически проверяемо.

Через $Q_{i} \subseteq Q$ обозначим множество состояний а.-функции $f$, достижимых из начального состояния за $i$ тактов. Скажем, что а.-функция $f$ с уравнениями (1) имеет память в $j$-й момент, если для некоторого слова $\alpha \in E_{2}^{n}$ длины $j-1$ и некоторых букв $a, b, c \in E_{2}^{n}$

$$
\psi\left(\varphi\left(q_{1}, \alpha a\right), c\right) \neq \psi\left(\varphi\left(q_{1}, \alpha b\right), c\right),
$$


в противном случае скажем, что а.-функщия $f$ не имеет памяти в $j$-й момент. Обозначим множество всех а.-функщй, не имеющих памяти в $j$-й момент, через $U(j), j=0,1, \ldots$

Автоматная функция $G: E_{2}^{\infty} \rightarrow E_{2}^{\infty}$ с уравнениями

$$
q(1)=0, \quad q(t+1)=a(t), \quad b(t)=q(t),
$$

называется автоматной функщией задержки или задержкой. Известно, что

$$
\left[\left\{G, f_{\mathrm{sh}}\right\}\right]=P_{a} .
$$

Через $\lambda$ обозначим пустую букву. Пусть $p, q, r, s \in Q$, определим бинарные отношения 2, 0, 1 на множестве $Q \times Q$. Пары $((p, q),(r, s))$ связаны отношением 2, 0, 1 , если, соответственно, существуют $a, b$ такие, что

$$
((\varphi(p, a)=r) \&(\varphi(q, b)=s) \&(\psi(p, a) \neq \psi(q, a))),
$$

существует $a$ такое, что

$$
((\varphi(p, a)=r) \&(\varphi(q, a)=s) \&(\psi(p, a)=\psi(q, a)))
$$

существуют $a, b$ такие, что

$$
((\varphi(p, a)=r) \&(\varphi(q, b)=s) \&(\psi(p, a)=\psi(q, a)) \&(\psi(p, b)=\psi(q, b))) .
$$

Пусть

$$
\text { ALL }=2 \cup 0 \cup 1
$$

и функция $\eta: Q^{2} \times Q^{2} \rightarrow\{\lambda, 0,1,2\}$ определена равенствами

$$
\eta((p, q),(r, s))= \begin{cases}\lambda, & \text { если }((p, q),(r, s)) \notin \mathbf{A L L}, \\ 2, & \text { если }((p, q),(r, s)) \in \mathbf{2}, \\ 0, & \text { если }((p, q),(r, s)) \in \mathbf{0} \backslash \mathbf{2}, \\ 1, & \text { если }((p, q),(r, s)) \in \mathbf{1} \backslash\{0 \cup 2\} .\end{cases}
$$

Пусть $\overline{\max }$ обозначает максимум относительно порядка $\lambda \leqslant 1 \leqslant 0 \leqslant 2$, а $\max$ - относительно порядка $0 \leqslant 1 \leqslant 2 \leqslant \lambda$.

Обозначим $I_{f}$ полный ориентированный граф с помеченными ребрами и множеством вершин $Q \times Q$, и пусть $\eta-$ функция меток ребер. Граф $I_{f}$ назовем локальным информационным графом а.-функции $f$. Для

$$
X_{1}, X_{2} \in 2^{Q \times Q}, \quad X_{1} \neq \varnothing, \quad X_{2} \neq \varnothing,
$$

обозначим через $E\left(X_{1}, X_{2}\right) \subseteq 2^{Q \times Q \times Q \times Q}$ всякую систему ребер графа $I_{f}$ такую, что всякое $x_{1} \in X_{1}\left(x_{2} \in X_{2}\right)$ соединсно ребром из $E\left(X_{1}, X_{2}\right)$ с $x_{2} \in X_{2}\left(x_{1} \in X_{1}\right)$, множество всех таких систем обозначим через $\Upsilon\left(X_{1}, X_{2}\right)$.

Зададим функцию $\Theta: 2^{Q \times Q} \times 2^{Q \times Q} \rightarrow\{\lambda, 0,1,2\}$ для $X_{1} \neq \varnothing, X_{2} \neq \varnothing$ соотношением

$$
\Theta\left(X_{1}, X_{2}\right)=\overline{\max }_{\Upsilon\left(X_{1}, X_{2}\right)}\left(\max _{E \in \Upsilon\left(X_{1}, X_{2}\right)}(\eta(E))\right),
$$

для $X_{1}=\varnothing$ или $X_{2}=\varnothing$ положим $\Theta\left(X_{1}, X_{2}\right)=\lambda$. 
Полный ориентированный граф $\hat{I}_{f}$ с помеченными ребрами, множеством вершин $2^{Q \times Q}$ и функщей меток $\Theta$ назовем глобальным информационным графом а.-функции $f$. Если $q \in Q_{i}$, то цикл $\{(q, q)\} E_{1} X_{1} E_{2} X_{2} \ldots X_{l-1} E_{l}\{(q, q)\}$ в графе $\hat{I}_{f}$ (соответственно в $I_{f}$ ) назовем $i$-м циклом, здесь через $E_{1}, \ldots E_{l}$ обозначены ребра. Если отметки ребер $i$-го цикла таковы, что

$$
\begin{aligned}
& \Theta\left(E_{1}\right)=1, \quad \Theta\left(E_{2}\right)=2, \quad \Theta\left(E_{3}\right) \neq 1, \ldots, \Theta\left(E_{l}\right) \neq 1, \\
& \left(\eta\left(E_{1}\right)=1, \quad \eta\left(E_{2}\right)=2, \quad \eta\left(E_{3}\right) \neq 1, \ldots, \eta\left(E_{l}\right) \neq 1\right),
\end{aligned}
$$

то назовем его $i$-м информационным циклом в графе $\hat{I}_{f}$ (соответственно в $I_{f}$ ).

Справедлива следующая лемма.

Лемма 3. Для системы $\left\{f, f_{\mathrm{sh}}\right\}$ такой, что $\left[\left\{f, f_{\mathrm{sh}}\right\}\right] \supseteq \mathbf{K}$, соотношение

$$
\left[\left(\left\{f, f_{\mathrm{sh}}\right\}\right] \ni G\right.
$$

справедливо тогда и только тогда, когда

(1) $f \notin U(i)$ при всех натуральных $i$,

(2) найдется натуральное $N \leqslant 2^{|Q|^{2}}$ такое, что для всех $i>N$ в графе $\hat{I}_{f}$ имеется $i$-й информачионный чикл.

Пусть $Q_{p}^{t} \subseteq Q$ - множество состояний а.-функции $f$, достижимых из состояния $p$ за $t$ тактов, а $R_{l}$ - множество состояний, из которых достижимо состояние $l$. Введем обозначение $Q_{p l}^{t}=Q_{p}^{t} \cap R_{l}$, тогда

$$
Q_{p l}^{t+1}=\left\{\varphi(p, a) \mid a \in E_{2}^{m}, p \in Q_{p l}^{t}\right\} \cap R_{l} .
$$

Последовательность $Q_{p l}^{t}, t=1,2, \ldots$, является детерминированной, а значит, и периодической. Можно выбрать период и предпериод этой последовательности равным, для простоты, одному и тому же числу $\rho$ при всех $p, l \in Q$. Очевидна грубая оценка $\rho \leqslant\left(2^{|Q|}\right)$ ! Итак для любых $p, l \in Q$

$$
Q_{p l}^{t}=Q_{p l}^{t+\rho}, \quad t=1,2, \ldots
$$

Доказательство теоремы 1 . Случай конечной системы $M=\left\{f_{1}, f_{2}, \ldots, f_{k}\right\}$ сводится к случаю, когда $M$ состоит из одного элемента $M=\{f\}$. Ввиду периодичности последовательности $Q_{q_{1}}^{t}, t=1,2, \ldots$, проверка условия $f \notin U(i)$ сводится к перебору значений $i<2 \rho$ и всех путей длины, не большей $i+1$, проходящих по состояниям а.-функции $f$. Число таких путей не превосходит $\left(2^{n}\right)^{2 \rho}$. Общее число операций при переборе не превосходит $2 \rho 2^{2 n \rho}$, где $\rho<\left(2^{|Q|}\right)$ !. Таким образом, общее число операций не превосходит числа $2\left(2^{|Q|}\right) !\left(2^{2 n\left(2^{|Q|}\right) !}\right)$.

Согласно лемме 2 включение $\left[\left\{f, f_{\mathrm{sh}}\right\}\right]_{K} \supseteq \mathbf{K}$ алгоритмически проверяемо. Рассмотрим проверяемость условия (1) леммы 3. Ввиду периодичности последовательности $Q_{q_{1}}^{t}, t=1,2, \ldots$, проверка условия $f \notin U(i)$, как уже доказано, занимает не более $2\left(2^{|Q|}\right) !\left(2^{2 n\left(2^{|Q|}\right) !}\right)$ операций. Оценим размеры графа $\hat{I}_{f}$. Он имеет множество вершин мощности $R=2^{|Q|^{2}}$. Для проверки наличия $i$-го информационного цикла необходимо проверить простые пути из вершин, достижимых из вершины $\left\{\left(q_{1}, q_{1}\right)\right\}$ за $i+2$ такта. 
Число таких путей не превосходит $R$ !. Вместо проверки счетного числа значений $i$, достаточно проверить состояния из множеств $R_{i}$ достижимых за $i$ тактов вершин графа $\hat{I}_{f}$. Если $\rho, \rho$ - период и предпериод последовательности $R_{1}, R_{2}, \ldots$, то необходимо проверить вершины из множеств $R_{1}, R_{2}, \ldots, R_{2 \rho \rho}$. Очевидно, что $\rho \rho \leqslant\left(2^{R}\right)$ !. Таким образом, общее число операций при проверке условия (2) леммы 3 не превосходит числа $\left(2^{2^{|Q|^{2}+1}}\right)$ !. Теорема доказана.

\section{4. Доказательства лемм}

Доказательство леммы 1. Докажем необходимость. Пусть $N \neq \rho$ и через а.-функции $f_{\mathrm{sh}}, f$ выражена а.-функция $g$, равная $B_{0, N}$. Это значит, что найдутся $k, D, s=D+k N$ такие, что а.-функция $g$ не является $(j, i)$-зависимой при $i \neq j(\bmod N)$. Зафиксируем для определенности $i, j, i<j(\bmod N)$.

Пусть а.-функция $g$ получена операцией обратной связи из а.-функция $\tilde{g}$. Покажем, что $\tilde{g}$ также не является $(j, i)$-зависимой. В самом деле, пусть эксперимент (2), получившийся из эксперимента

$$
((a(1), e(1)),(b(1), e(1))), \ldots((a(s), e(s)),(b(s), e(s)))
$$

нарушил $(j, i)$-зависимость. Это означает, что $b=\psi(q(j), a(i)) \neq b(i)$. Пусть входная буква $a(i)$ в диаграмме $g$ в состоянии $q(j)$ получилась из входной буквы $(a(i), e)$ в диаграмме $\tilde{g}$ в состояния $q(j)$. Тогда

$$
\begin{aligned}
\psi_{\tilde{g}}(q(j),(a(i), e)) & =(b, e), \\
\psi_{\tilde{g}}(q(j),(a(i), e(i))) & =\left(b^{\prime}, e^{\prime}\right) .
\end{aligned}
$$

По правилу применимости обратной связи $e=e^{\prime}$, и если есть сохранение $(j, i)$-зависимости для $\tilde{g}$, то $e^{\prime}=e(i)$, откуда следует, что $b^{\prime}=b=b(i)$, а это означает сохранение $(j, i)$-зависимости для $g$, что противоречит нашему предположению.

Пусть теперь $g$ получена суперпозицией а.-функций $g_{1}$ и $g_{2}$ и эксперимент (2) получен из экспериментов

$$
(a(1), c(1)), \ldots,(a(s), c(s)),(c(1), b(1)), \ldots,(c(s), b(s))
$$

а.-функций $g_{1}$ и $g_{2}$, соответственно. Если предположить, что $g_{1}$ и $g_{2}$ сохраняют $(j, i)$-зависимость, то получим, что $g$ также сохраняет $(j, i)$-зависимость. Значит, хотя бы одна из а.-функций $g_{1}$ или $g_{2}$ не сохраняет $(j, i)$-зависимость на $(D, s)$-экспериментах. Очевидно, что а.-функция $f_{\text {sh }}$ сохраняет $(j, i)$-зависимости на $(D, s)$ экспериментах.

Повторяя эти рассуждения, мы получим, что сама а.-функция $f$ не сохраняет $(j, i)$ зависимость на $(D, s)$ экспериментах при $i \neq j(\bmod N)$.

Если для $(D, s)$-экспериментов выполнена $(j, i)$-зависимость, то будет выполнена $\left(j_{1}, i\right)$-зависимость, где $j_{1}=j(\bmod \rho), j_{1} \neq i(\bmod N)$. В самом деле, это следует из того, что для каждого $p \in Q_{D}$ и для каждого $r \in Q_{p, p}^{i-D}$ справедливо равенство $Q_{r, p}^{j-i}=Q_{r, p}^{j_{1}-i}$. Так как $N \neq \rho$, для $i=j(\bmod N)$ а.-функция $f$ также не будет $(j, i)$-зависимой. Получилось, что счетчик $B_{D, N}$ допустим системой $\left\{f_{\mathrm{sh}}, f\right\}$. Очевидно, что из допустимости $B_{0,2 \rho}$ следует допустимость $B_{0, \rho}$. Необходимость доказана.

Докажем достаточность. Для фиксированных $N, D, k, s=D+k N$ и некоторого $j$ рассмотрим множество всех $(D, s)$ экспериментов $P$ с а.-функцией $f$, допускающее счетчик 
$B_{D, N}$. Пусть $|P|=t$ и $g_{j}$ есть параллельное соединение $t$ копий а.-функции $f$. Пусть $g_{j}$ описывается уравнениями (1) и $\alpha_{l} \in P-l$-й эксперимент из $P$,

$$
\begin{aligned}
\alpha_{l} & =\left(a^{(l)}(1), b^{(l)}(1)\right)\left(a^{(l)}(2), b^{(l)}(2)\right), \ldots,\left(a^{(l)}(s), b^{(l)}(s)\right), & & l=1,2, \ldots, t, \\
a(i) & =\left(a^{(1)}(i) \ldots a^{(t)}(i)\right), b(i)=\left(b^{(1)}(i) \ldots b^{(t)}(i)\right), & & i=1,2, \ldots, s .
\end{aligned}
$$

Можно считать, что среди $a(1), a(2), \ldots, a(s)$ нет равных (в противном случае можно добавить фиктивные входы и выбрать $a(1), a(2), \ldots, a(s)$ попарно не равными. Рассмотрим $(D, s)$-эксперимент (2) с а.-функцией $g_{j}$. Для $a \in E_{2}^{n}$ определим булеву векторфункцию $h_{j}: E_{2}^{m+n} \rightarrow E_{2}^{m}$, полагая

$$
h_{j}(x, y)= \begin{cases}a(i) & \text { для } x=a(i), y=b(i), i=1, \ldots, s, \\ a(j) & \text { для } y=\psi(q(j), x), x \in E_{2}^{n}, y \in E_{2}^{m} .\end{cases}
$$

Это можно сделать ввиду отсутствия $(j, i)$-зависимостей. В состоянии $q(j)$ выходная функция а.-функции $f_{j}(x)=h_{j}\left(x, g_{j}(x)\right)$ принимает значение $a(j)$ для всех входных букв, то есть является константой.

Рассмотрим а.-функцию

$$
F_{N, D, k}(x)=f_{s}\left(\ldots f_{2}\left(f_{1}(x)\right) \ldots\right)
$$

с начальным состоянием $(q(1), q(1), \ldots, q(1))$, она имеет $n$ входов и $n$ выходов. В состоянии $(q(j), q(j), \ldots, q(j))$ реализуется константа $a(j)$. После $n$ применений операции обратной связи от соответствующих выходов к соответствующим входам получится а.-функция, с точностью до булевой перекодировки, равная $B_{D, N}$. Достаточностьь доказана.

Доказательство леммы 2. Пусть $D<D+\rho<i<i+\rho<j<s-\rho<s$. Если для $(D, s)$ экспериментов выполнена $(j, i)$-зависимость, то для $\left(D_{1}, s_{1}\right)$ экспериментов, будет выполнена $\left(j_{1}, i_{1}\right)$-зависимость, где

$$
D_{1}<D_{1}+\rho<i_{1}<D+2 \rho<j_{1}<s_{1}<j_{1}+\rho,
$$

и $D_{1}, D ; i_{1}, i ; j_{1}, j ; s_{1}, s$ попарно совпадают по модулю $\rho$. Это следует из того, что для каждого $p \in Q_{q_{1}, p}^{D}$

$$
Q_{q_{1}, p}^{D}=Q_{q_{1}, p}^{D_{1}} \quad Q_{p, p}^{i}=Q_{p, p}^{i_{1}}
$$

для каждого $r \in Q_{p, p}^{i-D}$

$$
Q_{r, p}^{j-i}=Q_{r, p}^{j_{1}-i_{1}}
$$

и для каждого $l \in Q_{r, p}^{j-i}$

$$
Q_{l, p}^{s-j}=Q_{l, p}^{s_{1}-j_{1}}
$$

В случае другого расположения чисел $D, i, j$ мы таким же способом сократим интервалы между числами $1, D, i, j$. Следовательно, наличие или отсутствие $(j, i)$-зависимости на $(D, s)$-экспериментах равносильно, соответственно, наличию или отсутствию $\left(j_{1}, i_{1}\right)$ зависимости на $\left(D_{1}, s_{1}\right)$-экспериментах при $D_{1} \leqslant s_{1} \leqslant 5 \rho$. Последний факт проверяется перебором всех $\left(D_{1}, s_{1}\right)$-экспериментов.

Таким образом, условие выразимости системой $\left\{f_{\text {sh }}, f\right\}$ всех константных а.-функций алгоритмически проверяемо. Лемма 2 доказана. 
Пусть $I_{1}, I_{2}$ - полные графы с множествами вершин $P_{1}, P_{2}$ и функциями меток ребер

$$
\begin{aligned}
& \chi_{1}: P_{1} \times P_{1} \rightarrow\{\lambda, 0,1,2\}, \\
& \chi_{2}: P_{2} \times P_{2} \rightarrow\{\lambda, 0,1,2\},
\end{aligned}
$$

соответственно. Полный граф с множеством вершин $P_{1} \times P_{2}$ и функцией меток

$$
\chi:\left(P_{1} \times P_{2}\right) \times\left(P_{1} \times P_{2}\right) \rightarrow\{\lambda, 0,1,2\},
$$

где

$$
\chi\left(\left(\left(p_{1}{ }^{1}, p_{1}{ }^{2}\right),\left(p_{2}{ }^{1}, p_{2}{ }^{2}\right)\right)\right)=\overline{\max }\left(\chi_{1}\left(\left(p_{1}{ }^{1}, p_{2}{ }^{1}\right)\right), \chi_{2}\left(\left(p_{1}{ }^{2}, p_{2}{ }^{2}\right)\right),\right.
$$

назовем прямым произведением графов $I_{1}, I_{2}$ и обозначим $I_{1} \times I_{2}$. Граф $I \times \ldots \times I$ (c $l$ сомножителями) будем называть $l$-й степенью графа $I$ и обозначать через $I^{l}$.

Предложение 1. В графе $\hat{I}_{f}$ существует $i$-й информационный цикл тогда и только тогда, когда для некоторого натурального $l \leqslant 2^{n}|Q|^{2} 2^{|Q|^{2}}$ существует $i$-й информачионный чикл в графе $\left(I_{f}\right)^{l}$.

Доказательство. Пусть $f$ - а.-функция, определенная уравнениями (1), $I_{f}$ - ее локальный информационный граф, $\hat{I}_{f}-$ ее глобальный информационный граф, $q \in Q_{i}$, $X_{0}=\{(q, q)\}$,

$$
X_{0} \stackrel{x_{0}}{\longrightarrow} X_{1} \stackrel{x_{1}}{\longrightarrow} \ldots \stackrel{x_{s-2}}{\longrightarrow} X_{s-1} \stackrel{x_{s-1}}{\longrightarrow} X_{0}
$$

- $i$-й информационный цикл в $\hat{I}_{f}$. Длина $s$ этого цикла не превосходит числа вершин графа $\hat{I}_{f}$, которое не превосходит $2^{|Q|^{2}}$. Пусть

$$
\Upsilon_{j}=\Upsilon\left(X_{j}, X_{j+1}\right), \quad j=0,1, \ldots, s-1,
$$

- система ребер графа $I_{f}$, по которым выбрана метка $x_{j}=\max _{E \in \Upsilon_{j}} \chi(E)$ ребра $X_{j}, X_{j+1}$ графа $\hat{I}_{f}$. Пусть $l$ - число разных цепей, идущих по ребрам систем $\Upsilon_{j}, j=0,1, \ldots, s-1$, из $X_{0}$ в $X_{0}$, тогда для некоторого $l$ в $\left(I_{f}\right)^{l}$ существует $i$-й информационный цикл, составленный из указанных цепей. Заметим, что

$$
l \leqslant|Q|^{2} 2^{n} s=2^{n}|Q|^{2} 2^{|Q|^{2}} .
$$

Докажем обратное утверждение. Пусть в $\left(I_{f}\right)^{l}$ имеется $i$-й информационный цикл, $q \in Q_{i}$

$$
\left(p_{1}^{0}, \ldots, p_{l}^{0}\right) \stackrel{x_{0}}{\longrightarrow} \ldots \stackrel{x_{s-2}}{\longrightarrow}\left(p_{1}^{s-1}, \ldots, p_{l}^{s-1}\right) \stackrel{x_{s-1}}{\longrightarrow}\left(p_{1}^{0}, \ldots, p_{l}^{0}\right),
$$

$p_{j}^{r} \in Q \times Q, r=1, \ldots, s-1, j=1, \ldots, l,\left(p_{1}^{0}, \ldots, p_{l}^{0}\right)=((q, q), \ldots,(q, q))$. Пусть

$$
X_{i}=\left\{q_{j}^{i} \mid j=1,2, \ldots, l\right\}
$$

тогда цикл

$$
\left(X_{0}\right) \stackrel{y_{0}}{\longrightarrow}\left(X_{1}\right) \stackrel{y_{1}}{\longrightarrow} \ldots \stackrel{y_{s-1}}{\longrightarrow}\left(X_{0}\right)
$$

будет глобальным информационным циклом в $\hat{I}_{f}$. В самом деле, цепочка $y_{0}=x_{0}=1$, $y_{1}=x_{1}=2$ не может измениться, так как она уже максимальна. Если $x_{j}=0$ и возникла ситуация, когда для одной системы ребер метка равна 0 , а для другой 1 , то функция $\overline{\max }$ выберет метку 0 . Метка $\lambda$ возникнуть здесь не может.

Предложение доказано. 


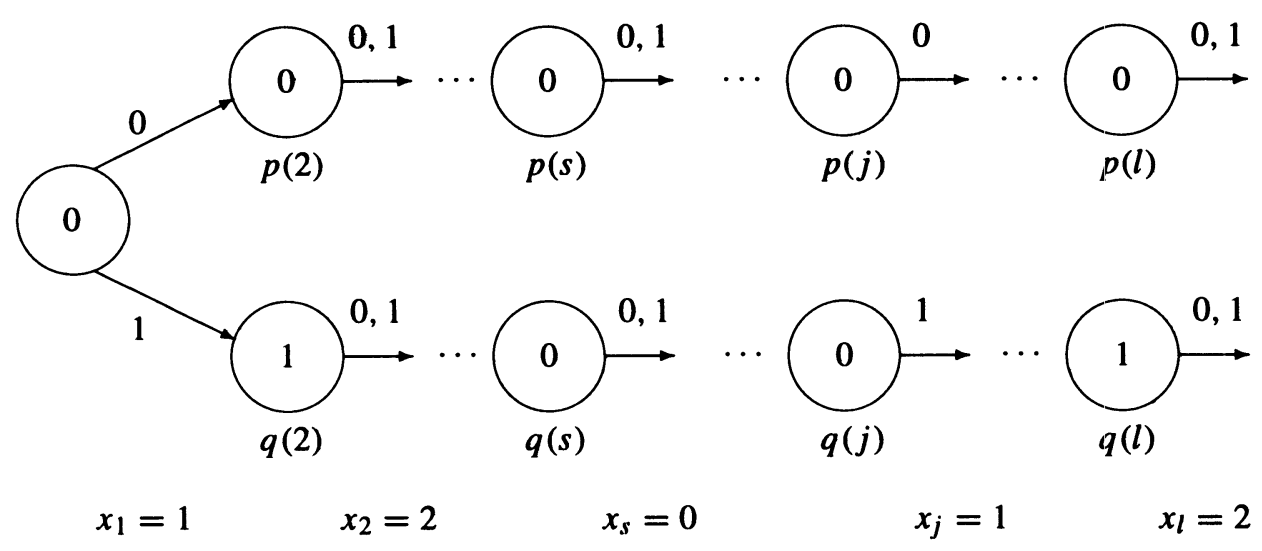

Рис. 1.

Пусть $g(x)=y$ - одноместная а.-функция с уравнениями (1), $I_{g}-$ ее локальный информационный граф. Путь

$$
(p(1), q(1)) \stackrel{x_{1}}{\longrightarrow}(p(2), q(2)) \stackrel{x_{2}}{\longrightarrow} \ldots \stackrel{x_{l-1}}{\longrightarrow}(p(l), q(l))
$$

в графе $I_{g}$, для меток которого и функций $\varphi$ и $\psi$ выполнено соотношение (5), назовем приведенным (см. рис. 1).

Здесь $\psi_{p}(a)=\psi(p, a)$ и

$$
\begin{aligned}
\psi_{p(i)} & \equiv \psi_{q(i)} \equiv 0, & & \text { при } x_{i} \neq 2, \\
\psi_{p(i)} & \equiv 0, \psi_{q(i)} \equiv 1, & & \text { при } x_{i}=2, \\
\varphi(p(i), 0) & =\varphi(p(i), 1)=p(i+1), & & \text { при } x_{i} \neq 1, \\
\varphi(q(i), 0) & =\varphi(q(i), 1)=q(i+1), & & \text { при } x_{i} \neq 1, \\
\varphi(p(i), 0) & =p(i+1), \varphi(q(i), 1)=q(i+1) & & \text { при } x_{i}=1 .
\end{aligned}
$$

Предложение 2. Пусть в графе $I_{f}$ есть $i$-й иикл длины $l$ с метками $x_{1}, \ldots, x_{l}$, тогда через $\left\{f, f_{\mathrm{sh}}\right\} \cup \mathbf{K}$ выразима одноместная а.-функция $g$, в графе $I_{g}$ которой имеется $i$-й приведенный цикл с теми же метками $x_{1}, \ldots, x_{l}$.

Доказательство. Пусть указанный цикл в графе $I_{f}$ имеет вид, приведенный на рис. 2 . Состояния $p_{2}, p_{3}, \ldots p_{l}$ образуют верхнюю ветвь, а состояния $q_{2}, q_{3}, \ldots q_{l}$ - нижнюю ветвь цикла,

$$
p_{i}, q_{i} \in Q, \quad a_{i}, b_{i} \in E_{2}^{n}, \quad c_{i}, d_{i} \in E_{2}^{m}
$$

Определим функщии

$$
\begin{aligned}
\gamma_{j}: E_{2} & \rightarrow E_{2}^{n}, \\
\delta_{j}: E_{2}^{n} \times E_{2}^{m} & \rightarrow E_{2}
\end{aligned}
$$



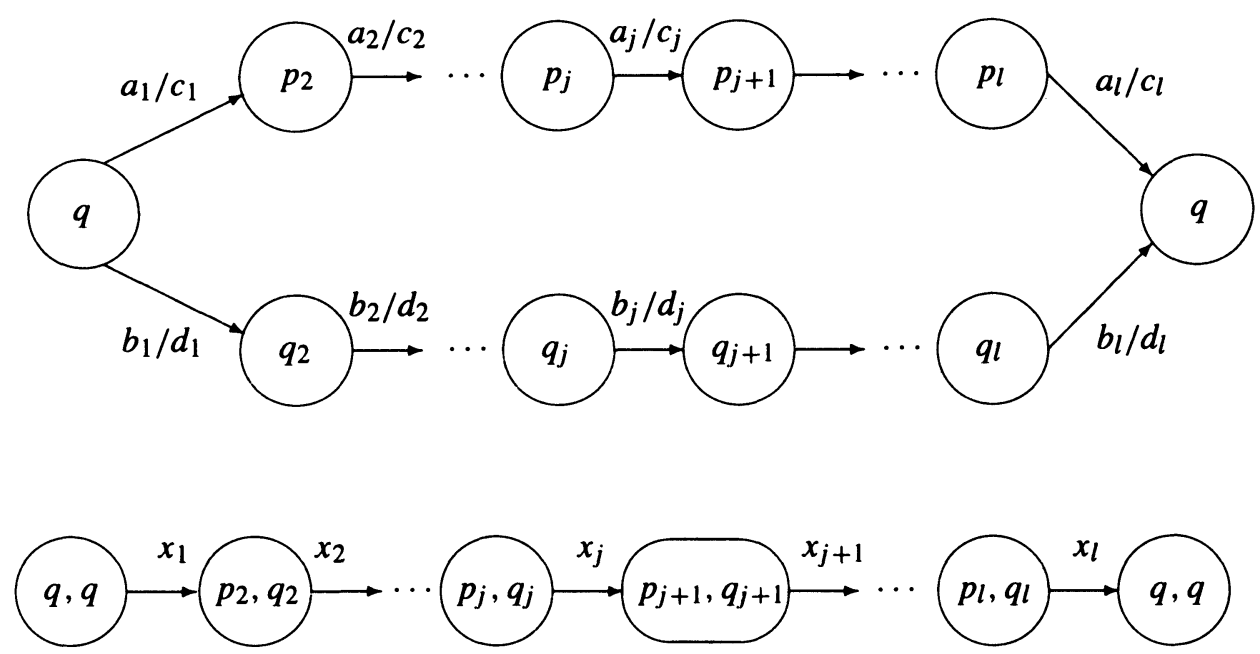

\section{Puc. 2.}

по $a_{j}, b_{j}, c_{j}, d_{j}$ (см. рис. 1$), j=1,2, \ldots l$, следующим образом:

$$
\begin{aligned}
& \gamma_{j}\left(z_{1}\right)=\left\{\begin{array}{l}
a_{j} \quad \text { при } x_{j}=0 ; \\
a_{j} \quad \text { при } x_{j}=1, z_{1}=0, \\
b_{j} \quad \text { при } x_{j}=1, z_{1}=1, \\
a_{j} \quad \text { при } x_{j}=2, a_{j}=b_{j}, c_{j} \neq d_{j}, \\
a_{j} \quad \text { при } x_{j}=2, z_{1}=0, \\
b_{j} \quad \text { при } x_{j}=2, z_{1}=1,
\end{array}\right. \\
& \delta_{j}\left(z_{2}, y_{1}\right)= \begin{cases}0 & \text { при } x_{j}=0, x_{j}=1, \\
0 & \text { при } x_{j}=2, a_{j}=b_{j}, c_{j} \neq d_{j}, z_{2}=c_{j}, y_{1}=a_{j}, \\
1 & \text { при } x_{j}=2, a_{j}=b_{j}, c_{j} \neq d_{j}, z_{2}=d_{j}, y_{1}=b_{j}, \\
0 & \text { при } x_{j}=2, a_{j} \neq b_{j}, \quad e_{j} \neq c_{j}, z_{2}=c_{j}, y_{1}=a_{j}, \\
1 & \text { при } x_{j}=2, a_{j} \neq b_{j}, e_{j} \neq c_{j}, z_{2}=e_{j}, y_{1}=a_{j}, \\
1 & \text { при } x_{j}=2, a_{j} \neq b_{j}, e_{j} \neq c_{j}, y_{1}=b_{j} .\end{cases}
\end{aligned}
$$

Пусть а.-функщии $D_{1}, D_{2} \in\left[\left\{\mathbf{K}, f_{\text {sh }}\right\}\right]$ суть функщии

$$
D_{1}: E_{2}^{*} \rightarrow\left(E_{2}^{n}\right)^{*}, \quad D_{2}:\left(E_{2}^{m} \times E_{2}^{n}\right)^{*} \rightarrow E_{2}^{*}
$$

$S_{1}, S_{2}$ - множества состояний а.-функций $D_{1}, D_{2}$ и $S_{1}=S_{2}=\{1,2, \ldots, l\}, z_{1} \in E_{2}$, $y_{1} \in E_{2}^{n}, z_{2} \in E_{2}^{m}, y_{2} \in E_{2}, s_{1} \in S_{1}, s_{2} \in S_{2}$, и функции $D_{1}, D_{2}$ задаются уравнениями

$$
\begin{aligned}
s_{1}(1) & =1, \\
s_{1}(t+1) & =s_{1}(t)+1 \quad(\bmod l),
\end{aligned}
$$



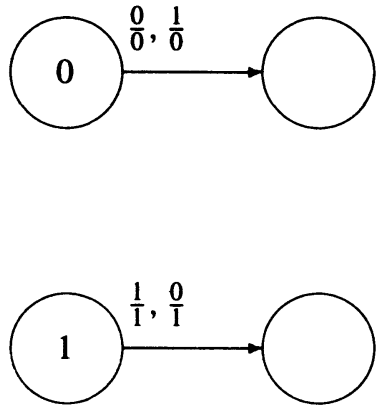

(a)
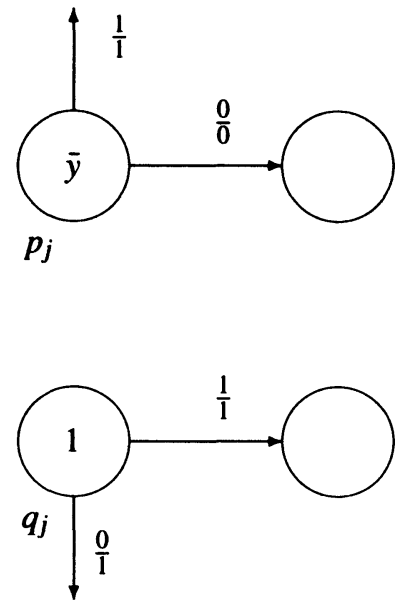

(b)

Pис. 3.

$$
\begin{aligned}
y_{1}(t) & =\gamma_{s_{1}(t)}\left(z_{1}\right), \\
s_{2}(1) & =1, \\
s_{2}(t+1) & =s_{2}(t)+1 \quad(\bmod l), \\
y_{2}(t) & =\delta_{s_{2}(t)}\left(z_{2}, y_{1}\right) .
\end{aligned}
$$

В графе а.-функции $h=D_{2}\left(f\left(D_{1}\left(z_{1}\right)\right), D_{1}\left(z_{1}\right)\right)$ имеется почти приведенный цикл с такими же отметками $x_{1}, \ldots x_{l}$ с той лишь разницей, что для $x_{i}=2$ могут быть два варианта переходов (a) и (b) (cм. рис. 3).

Далее мы сведем случай (b) к случаю (a). Пусть а.-функция

$$
H_{j}(u, v, z), \quad H_{j}:\left(E_{2}^{3}\right)^{*} \rightarrow E_{2}^{*}
$$

имеет множество состояний $S=\{1,2, \ldots, l\}$ и уравнения

$$
\begin{aligned}
s(1) & =1, \\
s(t+1) & =s(t)+1 \quad(\bmod l), \\
H_{j}(t) & = \begin{cases}u(t) & \text { для } s(t) \neq j, \\
u(t)+v(t)+z(t) & \text { для } s(t)=j .\end{cases}
\end{aligned}
$$

Если номер $j$ соответствует случаю (b), то а.-функция $F_{j}(y)=H_{j}(h(y), \overline{h(\bar{y})}, \bar{y})$ имеет фрагмент диаграммы, представленный на рис. 4, и приведенный цикл с теми же отметками $x_{1}, x_{2}, \ldots x_{l}$. В самом деле, для $s(t) \neq j$ выполнено соотношение $F_{j} \equiv h(y)$, а также условия (5). Если имеет место случай (b), то выходная функция в состоянии $\left(p_{j}, q_{j}\right)$ (см. рис. 4) равна $\bar{y}+0+\bar{y}=0$, в состоянии $\left(q_{j}, p_{j}\right)$ выходная функция равна $1+\bar{y}+\bar{y}=1$.

Таким образом, для $F_{j}(y)$ будут выполнены все условия (5), кроме условия

$$
\varphi(p(j), 1)=p(j+1), \quad \varphi(q(j), 0)=q(j+1)
$$




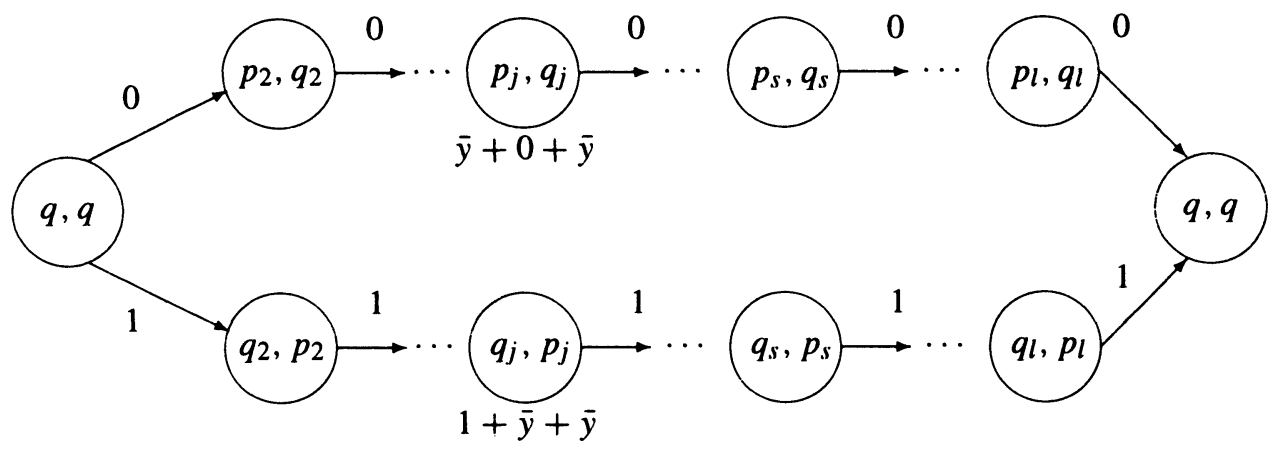

\section{Pис. 4.}

в случае (b). При помощи срабатывающей в $j$-й момент обратной связи указанный недостаток исправляется и фрагмент диаграммы приводится к виду, указанному на рис. 1 , схемой, изображенной на рис. 5 , где а.-функция $w_{j}(x, y)$

$$
w_{j}:\left(E_{2}^{2}\right)^{*} \rightarrow E_{2}
$$

имеет множество состояний $S$ и уравнения

$$
\begin{aligned}
s(1) & =1, \\
s(t+1) & =s(t)+1 \quad(\bmod l), \\
w_{j}(t) & = \begin{cases}x(t) & \text { для } s(t) \neq j, \\
y(j) & \text { для } s(t)=j .\end{cases}
\end{aligned}
$$

Предложение 2 доказано.

Предложение 3. Пусть а.-функция $f$ есть задержка $G$, тогда найдется натуральное $N \leqslant|Q|^{2}$ такое, что для любого $i>N$, в графе $I_{f}$ имеется $i$-й информачионный чикл.

Доказательство. Пусть $f$-одноместная а.-функция с уравнениями (1) и $f$ эквивалентна $G$. Последовательность $\left\{q_{1}\right\}, Q_{1}, Q_{2}, \ldots$ имеет предпериод $\rho$ и период $\rho$. Пусть $i>\rho$ и $q \in Q_{i}$ - такое состояние, что для любого слова $\beta \in E_{2}^{*}$ найдется слово $\gamma \in E_{2}^{*}$ такое, что $\varphi(q, \beta \gamma)=q\left(q-\right.$ состояние из сильно связной компоненты). Пусть слова $\alpha, \bar{\alpha} \in E_{2}^{*}$ таковы, что

$$
\alpha=a_{1} a_{2} \ldots a_{\rho}, \quad \bar{\alpha}=\overline{a_{1} a_{2}} \ldots \overline{a_{\rho}} .
$$

Рассмотрим путь (см. рис. 6) в графе $I_{f}$. В состояние $p_{j}$ из $q$ ведет путь $a_{1} a_{2} \ldots a_{j-1}$, в состояние $q_{j}-$ путь $\bar{a}_{1} \bar{a}_{2} \ldots \bar{a}_{j-1}$.

Так как для а.функции $f=G$ в состоянии $p_{j}$ реализуется константа $a_{j-1}$, а в состоянии $q_{j}$ константа $\bar{a}_{j-1}$, получаем, что $x_{1}=1, x_{2}=x_{3}=\ldots=x_{\rho}=2$. Из того, что $Q_{i}=Q_{i+\rho}$, следует, что $p_{\rho+1}, q_{\rho+1} \in Q_{i}$. Для натурального $s$ рассмотрим множество

$$
H_{s}=\left\{\alpha^{s-l}(\bar{\alpha})^{l} \mid l=1,2, \ldots, s\right\} \subset E_{2}^{*}
$$

слов длины $s \rho$, составленных из слов $\alpha$ и $\bar{\alpha}$. Пусть $\gamma, \beta \in H_{s}$ таковы, что

$$
\gamma=\alpha^{l}(\bar{\alpha})^{s-l}, \quad \beta=\alpha^{j}(\bar{\alpha})^{s-j},
$$




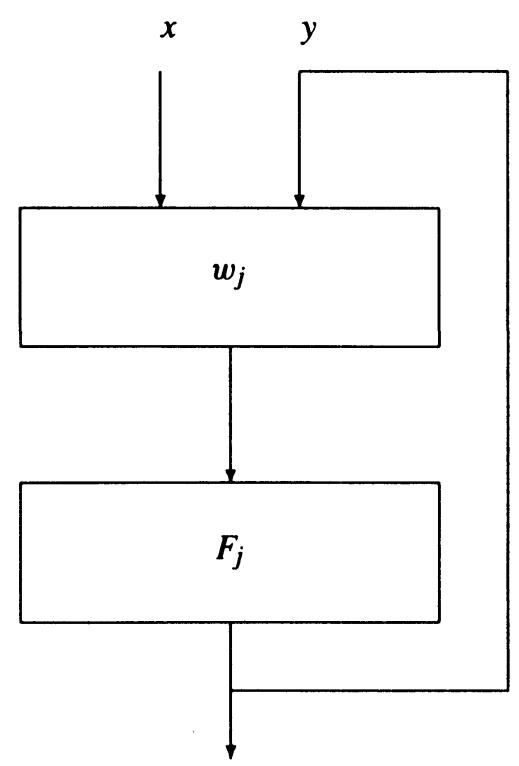

Pис. 5.

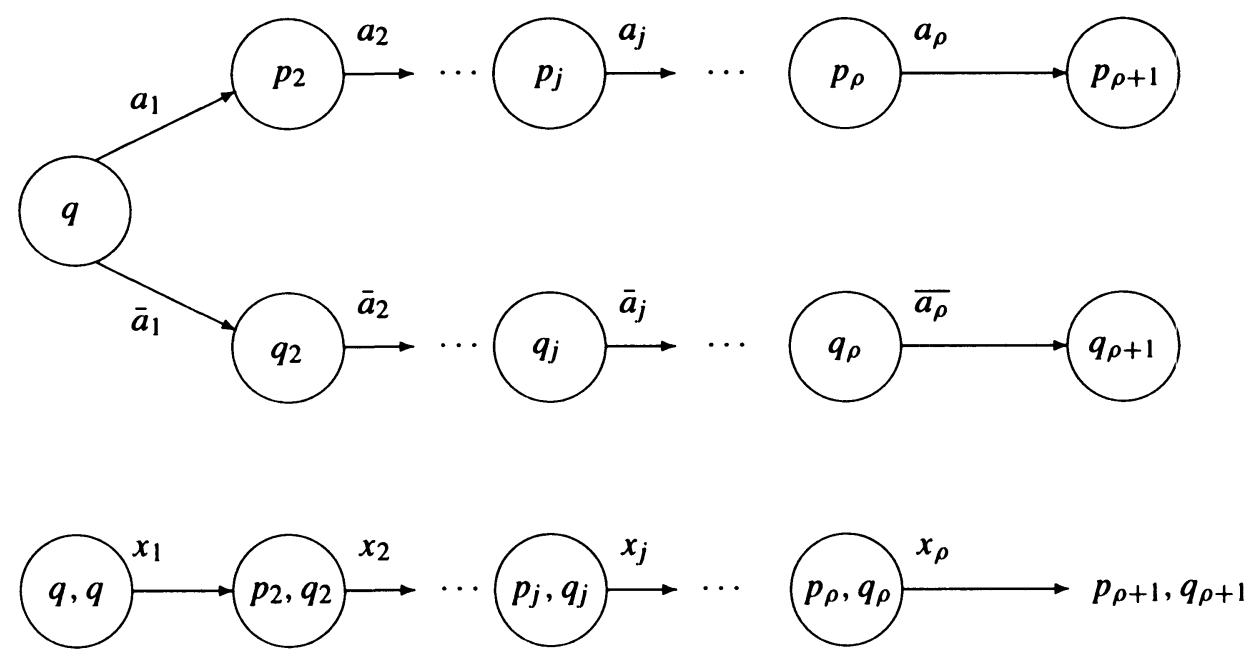

Рис. 6.

где $l<j$, состояния $r_{\beta}, r_{\gamma}, p \in Q$ такие, что

$$
r_{\beta}=\varphi(q, \beta), \quad r_{\gamma}=\varphi(q, \gamma), \quad p=\varphi\left(q, \alpha^{l}\right) .
$$

В графе $I_{f}$ из пары $(q, q)$ в пару $\left(r_{\beta}, r_{\gamma}\right)$ ведет путь (см. рис. 7$)$, определяемый парой 


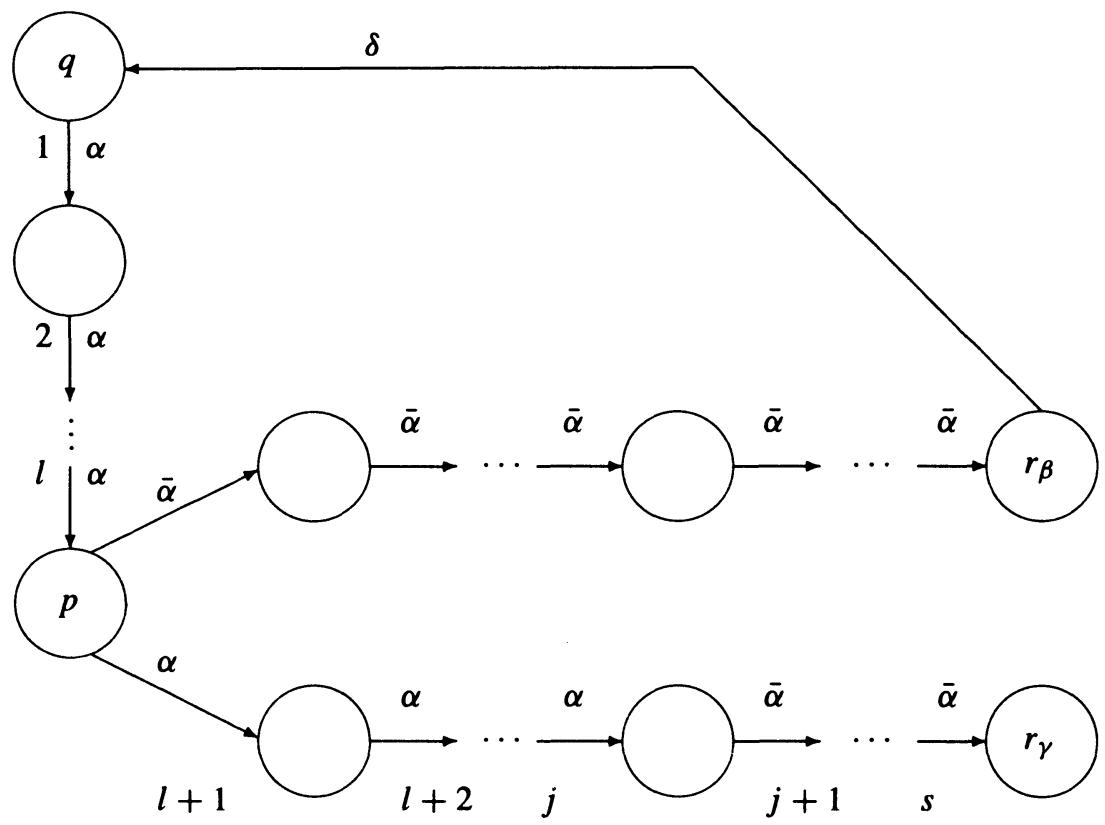

Рис. 7.

слов $(\beta, \gamma)$ с метками

$$
\begin{aligned}
& y_{1}=\ldots=y_{l \rho}=0, \quad y_{l \rho+1}=1, \\
& y_{l \rho+2}=\ldots=y_{j \rho+1}=2, \\
& y_{j \rho+2}=\ldots=y_{s \rho}=0 .
\end{aligned}
$$

Если предположить, что для любого $s$ и любых $\gamma, \beta \in H, \gamma \neq \beta$ справедливо неравенство $r_{\beta} \neq r_{\gamma}$, то получим противоречие с конечностью множества состояний а.функции $f$.

Следовательно, найдется такое $s$ и такие слова $\gamma, \beta \in H, \gamma \neq \beta$, длины $s \rho$, что справедливо равенство $r_{\beta}=r_{\gamma}$. Заметим, что $r_{\beta}, r_{\gamma}, p, q \in Q_{i}$. Пусть $\delta$ - такое слово, что $\varphi\left(r_{\beta}, \delta\right)=q$, тогда из пары $(p, p)$ начинается искомый $i$-й информационный цикл (см. рис. 7). Очевидно, что всякое состояние достижимо словом длины, не большей $|Q|^{2}$.

Предложение 3 доказано.

Предложение 4. Пусть а.-функция $h$ получается суперпозичией $а$.-функций $h_{1} u h_{2}, n p u$ этом в графах $I_{h}, I_{h_{1}}, I_{h_{2}}$, соответственно, есть ребра с метками

$$
\begin{gathered}
\left(\left(p_{1}, p_{2}\right),\left(q_{1}, q_{2}\right)\right) \stackrel{x}{\longrightarrow}\left(\left(r_{1}, r_{2}\right),\left(s_{1}, s_{2}\right)\right), \\
\left(p_{1}, q_{1}\right) \stackrel{y}{\longrightarrow}\left(r_{1}, s_{1}\right), \\
\left(p_{2}, q_{2}\right) \stackrel{z}{\longrightarrow}\left(r_{2}, s_{2}\right),
\end{gathered}
$$

тогда метки $x, y, z$ связаны соотномениями

$$
(x=2) \rightarrow((y=2) \vee(z=2)), \quad(x=0) \rightarrow((y=2) \vee(y=z=0)) .
$$




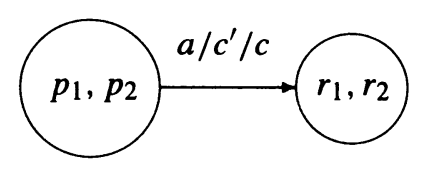

Рис. 8.
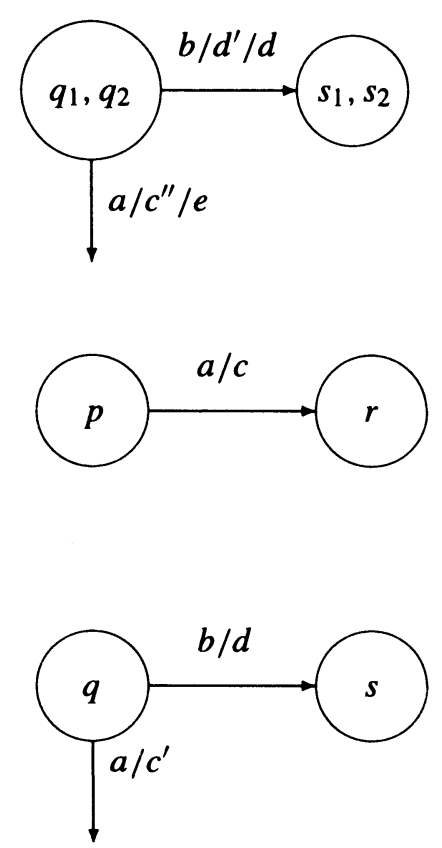

Рис. 9.

Доказательство. Рассмотрим фрагмент диаграммы а.-функщии $h$ (см. рис. 8), где показаны выходы а.-функщий $h_{1}, h_{2}$. Пусть $x=2$ и $c \neq e$, тогда, если $c^{\prime \prime} \neq c^{\prime}$, то $y=2$, если $c^{\prime \prime}=c^{\prime}$, то $z=2$. Пусть $x=0, a=b, c=d$, тогда при $c^{\prime} \neq d^{\prime}$ справедливо равенство $y=2$, а при $c^{\prime}=d^{\prime}-$ равенство $y=z=0$. Предложение 4 доказано.

Предложение 5. Пусть а.-функция $h$ получена из а.-функции $g$ при помощи операчии обратной связи и в графе $I_{h}$ есть ребро

$$
(p, q) \stackrel{x}{\longrightarrow}(r, s),
$$

тогда в графе $I_{g}$ есть ребро

$$
(p, q) \stackrel{y}{\longrightarrow}(r, s),
$$

и при этом метки ребер связаны соотношениями

$$
(x=2) \rightarrow(y=2), \quad(x=0) \rightarrow(y \neq 1) .
$$

Доказательство. Пусть а.-функция $h$ получена из а.-функции $g$ с уравнениями (1) операцией обратной связи, примененной от последнего выхода к последнему входу. На рис. 9 

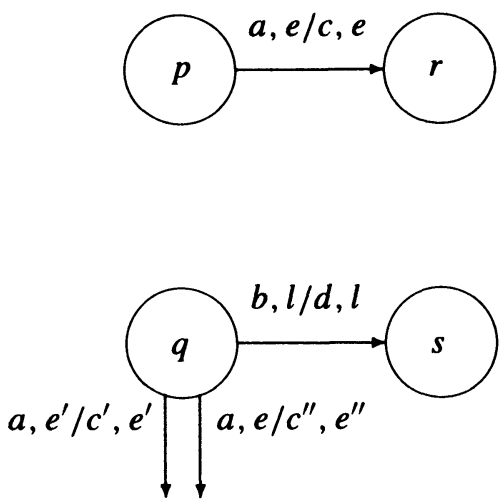

Рис. 10.
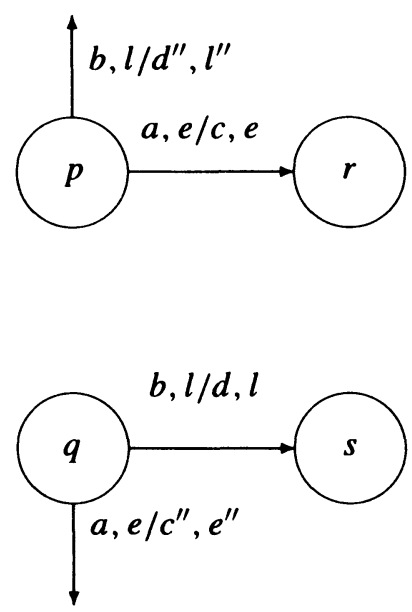

Рис. 11.

изображен фрагмент диаграммы а.-функции $h$, на рис. 10 - соответствующий ему фрагмент диаграммы а.-функции $g$, где $e, l, e^{\prime}, e^{\prime \prime} \in E_{2}, a, b \in E_{2}^{n-1}, c, d, c^{\prime}, c^{\prime \prime} \in E_{2}^{m-1}$.

Пусть $x=2, c^{\prime} \neq c$, и предположим противное, то есть предположим, что $y=2$, или $\left(c^{\prime \prime}, e^{\prime \prime}\right)=(c, e)$. Последний выход а.-функции $g$ не зависит от ее последнего входа, а первая компонента у букв $\left(a, e^{\prime}\right)$ и $(a, e)$ одна и та же, поэтому (см. рис. 10 , состояние $\left.q\right)$ справедливо равенство $e^{\prime \prime}=e^{\prime}=e$. Но тогда $\left(a, e^{\prime}\right)=(a, e)$ и это один и тот же входной сигнал, и следовательно, $\left(c^{\prime}, e^{\prime}\right)=\left(c^{\prime \prime}, e^{\prime \prime}\right)=(c, e)$. Получили противоречие с тем, что $c^{\prime} \neq c$, значит, $y=2$.

Пусть теперь $x=0, a=b, c=d$ (см. рис. 11); предположим противное, то есть предположим, что $y=1$ и $(a, e) \neq(b, e)$, тогда $e^{\prime \prime}=e, c^{\prime \prime}=c, d^{\prime \prime}=d, l^{\prime \prime}=l$. Так как (по определению обратной связи) последний выход не зависит от последнего входа, справедливо соотношение $e^{\prime \prime}=l, l^{\prime \prime}=e$, значит, $e=l$, откуда получаем противоречивое равенство $(a, e)=(b, l)$, значит, $y \neq 1$.

Предложение 5 доказано.

Пусть $g(x)=y$ - одноместная а.-функция с уравнениями (1), имеющая память в $j$-й 


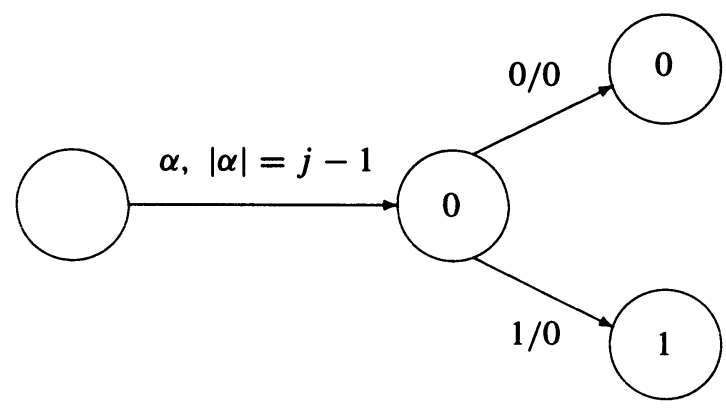

Рис. 12.

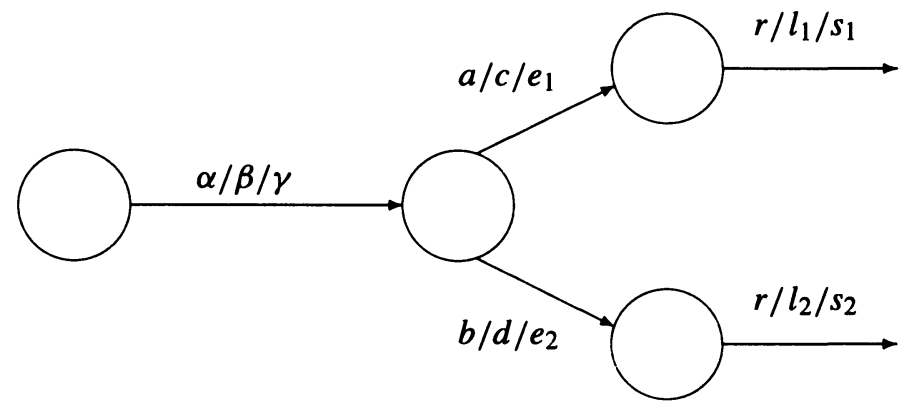

Рис. 13.

момент. Фрагмент диаграммы $g$ вида, приведенного на рис. 12 , назовем $j$-м приведенным фрагментом.

На рис. 12 в состояниях, помеченных 0,1 , реализуются выходные функции 0,1 , соответственно.

Предложение 6. Пусть а.-функция $h$ получается суперпозичией $а$.-функций $h_{1} u h_{2} u$ $h \notin U(i)$ дія некоторого $i$, тогда $h_{1} \notin U(i)$ или $h_{2} \notin U(i)$.

Доказательство. Рассмотрим фрагмент а.-функции $h$, приведенный на рис. 13 , на котором нарушается свойство $h \in U(i)$.

На рис. $13 s_{1} \neq s_{2}$. Если $l_{1} \neq l_{2}$, то $h_{1} \notin U(i)$; если $l_{1}=l_{2}$, то $h_{2} \notin U(i)$. Предложение 6 доказано.

Предложение 7. Пусть а.-функчия $h$ получена из а.-функчии g операчией обратной связи $u h \notin U(i)$ для некоторого $i$, тогда $g \notin U(i)$.

Доказательство. Пусть фрагмент а.-функции $h$ (см. рис. 3) получился при операции обратной связи из фрагмента а.-функции $g$ (см. рис. 14).

Здесь $d \neq d^{\prime}$. Если $e_{3}=e_{4}$, то $g \notin U(i)$. Предположим, что $e_{3} \neq e_{4}$, тогда по определению обратной связи $e_{3}=e_{3}^{\prime \prime}$ и, значит, по сигналу $\left(c, e_{4}\right)$ в состояниях $p$ и $r$ будут получаться разные выходные буквы $\left(d^{\prime \prime}, e_{3}\right)$ и $\left(d^{\prime}, e_{4}\right)$. Значит, $g \notin U(i)$.

Предложение 7 доказано. 


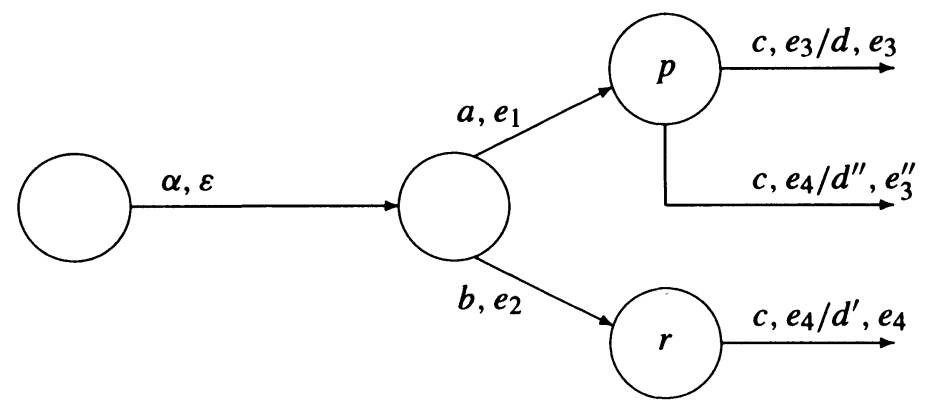

Рис. 14.

Зафиксируем $i>N$. Выберем в графе $\hat{I}_{f} i$-й информационный цикл длины $p$. По предложению 1 в графе $\left(I_{f}\right)^{l}$ для некоторого $l$ существует $i$-й информационный цикл длины $p, j$-я проекция которого имеет метки $x_{i j}^{1}, \ldots, x_{i j}^{p}, j=1,2, \ldots, l$, причем

$$
\begin{aligned}
& 2 \max _{j} x_{i j}^{1}=1, \quad \max _{j} x_{i j}^{2}=2, \\
& \max _{j} x_{i j}^{s} \neq 1, \quad s=3,4, \ldots, p .
\end{aligned}
$$

По предложению 2 найдется одноместная а.-функция $g_{i j} \in\left[\mathbf{K} \cup\left\{f, f_{\mathrm{sh}}\right\}\right]$, в информационном графе которой существует приведенный цикл с теми же метками $x_{i j}^{1}, \ldots, x_{i j}^{p}$, $j=1,2, \ldots, l$ (см. рлсс. 1). Пусть

$$
\left\{j \mid x_{i j}^{s}=1\right\}=\varepsilon_{i}(s), \quad 1 \leqslant s \leqslant p .
$$

Из определения информационного цикла следует, что при $s>2, \varepsilon_{i}(s) \neq \varnothing$, или при $s=2$ существует натуральное $\xi_{i}(s)$ такое, что $x_{i, \xi_{i}(s)}^{s}=2$. Рассмотрим схему, приведенную на рис. 15 , где $H_{1}$ и $H_{2}$ - булевы вектор-функции

$$
H_{1}\left(z_{1}, z_{2}, s\right)=\left(x_{1}, x_{2}, \ldots, x_{l}\right), \quad H_{2}\left(y_{1}, y_{2}, \ldots, y_{l}, s\right)=(u, v),
$$

в задании которых

$$
\begin{aligned}
& u= \begin{cases}y_{\xi_{i}(2)} & \text { при } s=2, \\
y_{\xi_{i}(s)} & \text { при } \varepsilon_{i}(s) \neq \varnothing, \\
0 & \text { при } \varepsilon_{i}(s)=\varnothing,\end{cases} \\
& v= \begin{cases}y_{\xi_{i}(2)} & \text { при } s=2, \\
0 & \text { при } s \neq 2,\end{cases} \\
& x_{j}= \begin{cases}z_{1} & \text { при } j=\xi_{i}(2), s=2, \\
z_{1} & \text { при } j \in \varepsilon_{i}(s), s>2, \\
z_{2} & \text { при } j \in \varepsilon_{i}(s), s=1, \\
0 & \text { в остальных случаях. }\end{cases}
\end{aligned}
$$

А.-функции $B_{0, p}, g_{i j}, j=1,2, \ldots, l,-$ муровские, следовательно, применима операция обратной связи. Каждая из а.-функций $g_{i j}$ имеет диаграмму, указанную на рис. 16, с 


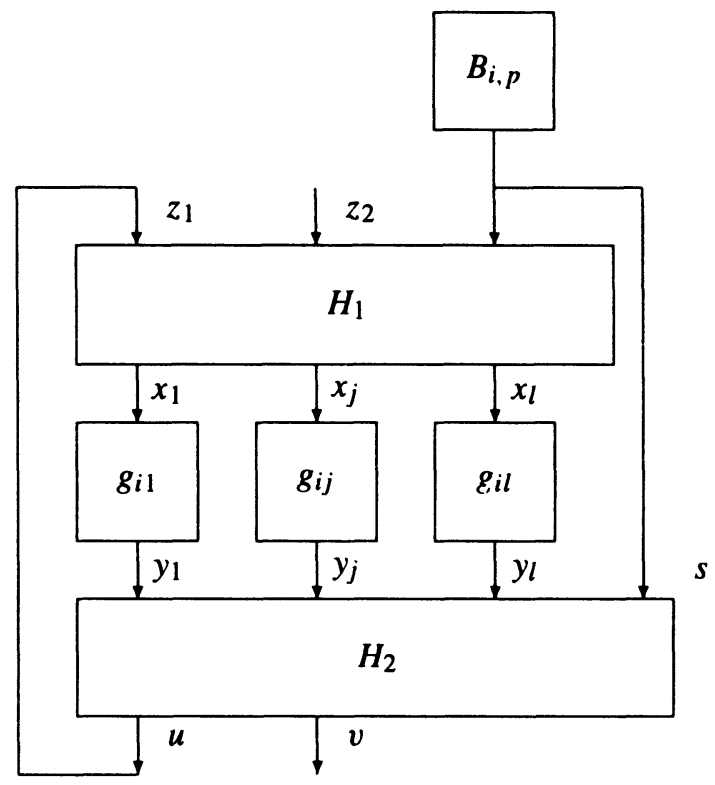

Рис. 15.

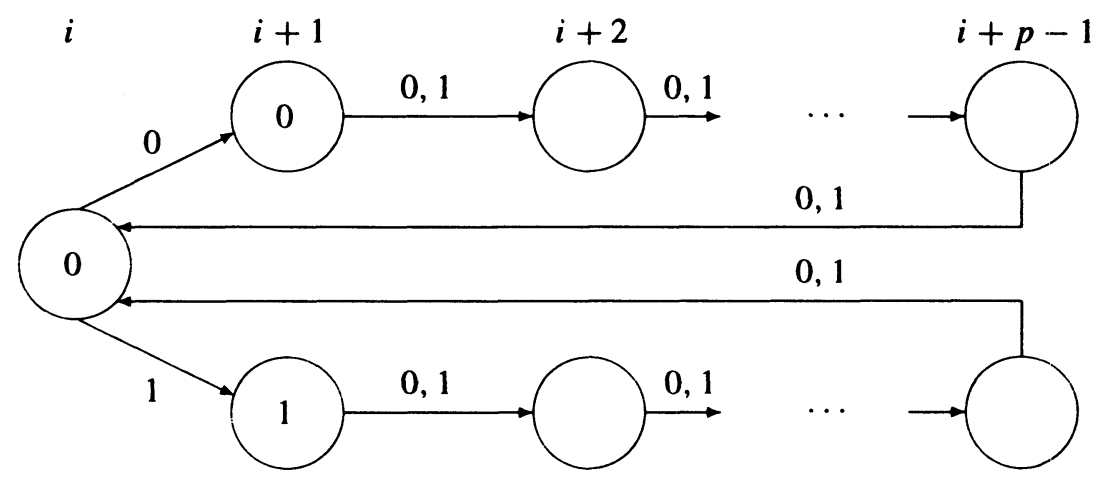

Рис. 16.

той лишь разницей, что для метки $x_{i j}^{s}=1$ переход не является безусловным. Схема на рис. 15 реализует а.-функцию $f_{p, i}$ с диаграммой, приведенной на рис. 16. Без ограничения общности можно выбрать длину цикла $p$, не зависящей от $i$. В самом деле, к каждой ветви $i$-го цикла можно добавить верхнюю ветвь, взятую произвольное число раз, тем самым, выбрать $p$ кратным длинам всех $i$-х информационных циклов, где $\rho \leqslant i \leqslant 2 \rho$. Для каждого $i<N$ по условию леммы $3 f \notin U(i)$, значит, в силу предложения 2 найдется а.-функция $g_{i} \in\left[\left\{f, f_{s h}, \mathbf{K}\right]\right)$, имеющая $i$-й приведенный фрагмент. Тогда схема на рис. 1 есть задержка $G$. Здесь а.-функция $D:\left(E_{2}^{N+p}\right)^{*} \rightarrow E_{2}^{*}$, имеет диаграмму, изображенную на рис. 18. Достаточность доказана.

Докажем необходимость. Пусть $\left[\left\{f, f_{\text {sh }}\right\}\right] \supseteq \mathbf{K}$ и $\left[\left\{f, f_{\text {sh }}\right\}\right] \ni G$. Покажем, что $f \notin U(i)$ для любого натурального $i$. В самом деле, $G \notin U(i)$, и если $G$ получена некоторой схемой $h$, последняя операция которой есть суперпозиция двух функций $h_{1}, h_{2}$, то по 


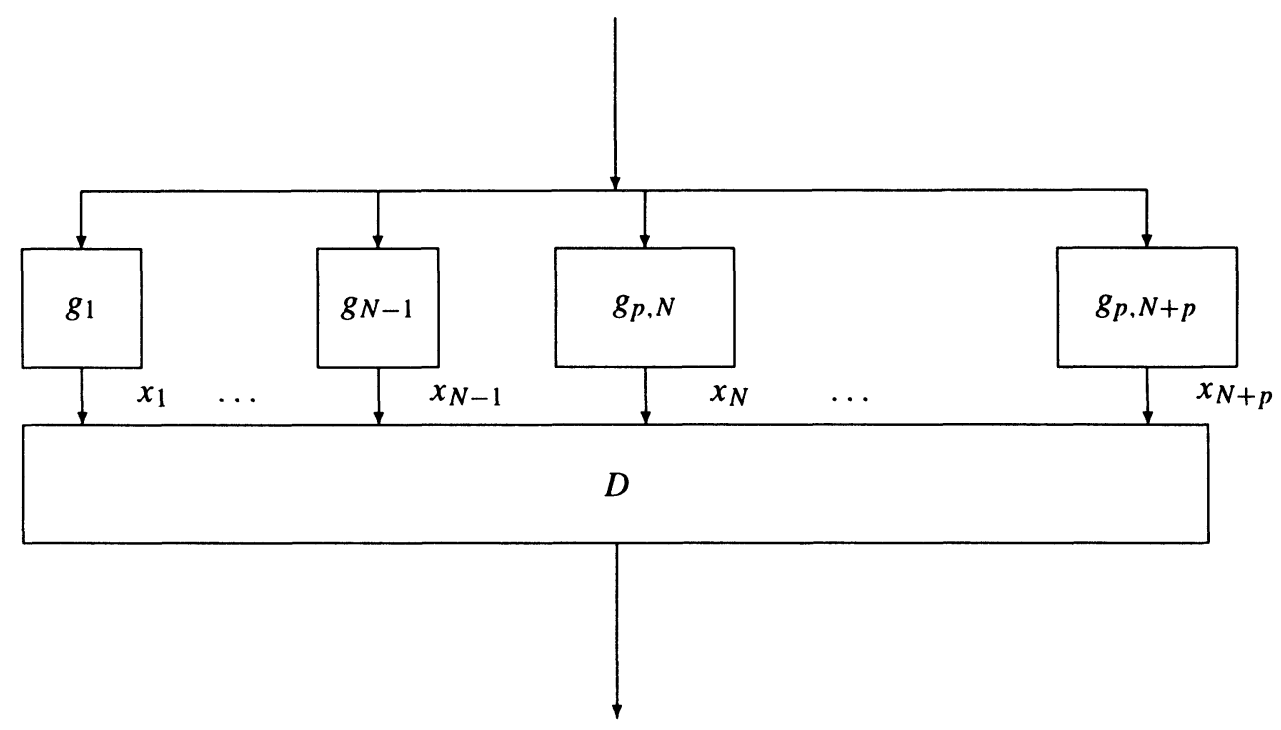

Pис. 17.

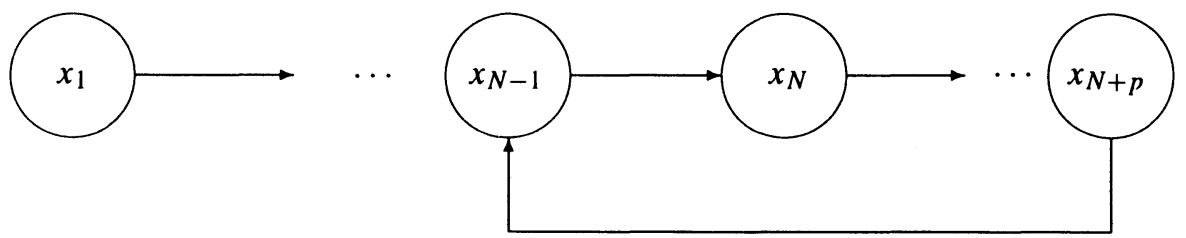

Рис. 18.

предложению $7 h_{1} \notin U(i)$ или $h_{2} \notin U(i)$. Если последняя операция указанной схемы операция обратной связи, то а.-функщия $g$, из которой получена $G$, по предложению 7 , обладает тем же свойством: $g \notin U(i)$. Применяя эти рассуждения к одной из подходящих а.-функций $g, h_{1}, h_{2}$, мы через конечное число шагов (равное числу операций в указанной схеме) придем к тому что $f \notin U(i)$, потому что $f_{\text {sh }} \in U(i)$, при всех $i$.

По предложению 3 найдется такое $N$, что в схеме $h$, где $h=g$, для любого $i>N$ в графе $I_{h}$ имеется $i$-й информационный цикл. Если $h$ получилась операцией суперпозиции из а.-функций $h_{1}, h_{2}$, то согласно предложению 4 в графе $I_{h_{1}} \times I_{h_{2}}$ имеется $i$-й информационный цикл. Если $h$ получилась из $g$ операцией обратной связи, то согласно предложению 5 , в $I_{g}$ имеется $i$-й информационный цикл. Поскольку в графе $I_{f_{\mathrm{sh}}}$ нет $i$-х информационных циклов при всех $i$, получаем, что $i$-й информационный цикл найдется в графе $\left(I_{f}\right)^{l}$ для некоторого $l$. По предложению 1 отсюда следует, что в графе $\widehat{I_{f}}$ также имеется $i$-й информационный цикл. Необходимость доказана. Лемма 3 доказана. 


\section{Список литературы}

1. Кудрявцев В. Б., О мощностях множеств предполных классов некоторых функциональных систем, связанных с автоматами. Докл. АН ССCP (1963) 151, №3, 493-496.

2. Кратко М. И., Алгоритмическая неразрешимость проблемы распознавания полноты для конечных автоматов. Докл. ДАН СССР (1964) 155, №1, 35-37.

3. Летичевский А. А., Условия полноты для конечных автоматов. Вычисл. матем. и матем. физика (1961), №4, 702-710.

4. Бабин Д. Н., Разрешимый случай задачи о полноте автоматных функций. Дискретная математика (1992) 4, №4, 41-56.

5. Бабин Д. Н., О разрешимости проблемы полноты для специальных систем автоматных функций. Дискретная математика (1996) 8, №4, 79-91.

6. Бабин Д. Н., Алгоритмическая разрешимость свойств полноты и $A$-полноты конечных систем автоматных функций с линейной истинностной частью. Интеллектуальные cucтемы (1998) 3, 51-69.

7. Бабин Д. Н., Конечность множества автоматных базисов Поста с разрешимой проблемой полноты. Дискретная математика (1998) 10, №3, 57-64.

Статья поступила 11.10 .2002$. 